\title{
Decreasing Serial Cost Sharing under Economies of Scale
}

\author{
M. Angeles de Frutos \\ Departamento de Economia, Universidad Carlos III, 28903 Getafe, Madrid, Spain \\ E-mail: frutos@eco.uc3m.es
}

We consider the problem of cost sharing in the presence of increasing returns to scale and potential strategic behavior on the part of consumers. We show that any smooth and strictly monotonic mechanism for which a Nash equilibrium exists for all profiles of convex and monotonic preferences must be dictatorial. However, we propose a cost sharing mechanism, the decreasing serial mechanism, for which an interesting domain restriction ensures existence of a noncooperative equilibrium for its cost sharing game. A characterization theorem of the mechanism based on the strategic properties of existence, uniqueness, and efficiency of its noncooperative equilibrium is provided. Journal of Economic Literature Classification Numbers: C70, C72, D70.

\section{INTRODUCTION}

Cost allocation is a practical problem that organizations of all kinds face. Universities allocate computing costs among departments. Aviation authorities set landing fees for aircraft based on their size. Regulatory commissions set rates for electricity, water, and other utilities based on the costs of providing these services. The common feature in all of these examples is that prices are not determined externally by market forces, but are set internally by mutual agreement or administrative decision.

A simple situation where a cost sharing problem can emerge is when a set of users share a technology that transforms a single input into a single output. We study this cost sharing problem when the cost function is increasing and concave. Examples include the division of costs incurred in setting telephone lines or a water supply system, the assessment of fees to pay for a secretary or a gardener's services, and so on. The situations we have in mind are those of cooperative production or natural nonregulated monopolies. For either of these situations there is no obvious answer to the question of how to divide the cost of producing the total output among the users. 
The cost sharing literature provides several examples of mechanisms for the allocation of a common cost. In particular, Moulin and Shenker [5] introduced the idea of serial cost sharing. They proposed a mechanism which allocates a portion of the cost of total supply to each consumer in order of increasing demand. Their mechanism should only be used when the consumption of every user is easily monitorable since it is not immune to secret pools of demands. Agents could benefit by, for instance, averaging their demands. The assumption that output and/or input cannot be freely transferred across agents gets rid of this possibility (see Moulin and Shenker [5]). When the cost function is convex and there exists potential strategic behavior on the part of consumers, this mechanism induces a game that is dominance solvable and whose unique equilibrium is a strong equilibrium. Moreover no other smooth cost sharing mechanism yields a unique Nash equilibrium at all preferences profiles.

Whenever the cost function $C$ is concave, the different users of the technology impose a positive externality on one another. One way to think of this externality is to identify it with the total surplus or savings, $\left(\sum_{i=1}^{n} C\left(q_{i}\right)-C\left(\sum_{i=1}^{n} q_{i}\right)\right)$, generated because of having more than one consumer demanding the good; this surplus should be shared among the agents in accordance with their contribution to it. Moulin and Shenker's mechanism allocates the largest surplus to the agent with the smallest demand. This agent contributes the least to the total surplus but she is the one that gets the largest savings.

We here propose a new mechanism allocating costs to consumers in decreasing order of demands. Our mechanism, which is intimately related to the one proposed by Moulin and Shenker, allocates the largest surplus to the agent with the greatest demand, since in some sense she creates the largest positive externality to the others. Moreover each consumer's share depends on her own demand and the demands of those who demand more than she does. Thus the mechanism generates a hierarchy in the decision making process. The first one in the hierarchy is the agent with the greatest demand; she is the one who pays relatively less and who affects all the others agents' payments with her demand. Since we go from the agent demanding the most to the one demanding the least we call this formula the decreasing serial rule. For purposes of comparison we will refer to Moulin and Shenker's mechanism as the increasing serial rule.

Our objective is to study the incentive properties of the decreasing serial rule when there exists potential strategic behavior on the part of consumers. We assume that each user of the technology has preferences over the possible vectors (quantity-payment), and that consumers choose their demands strategically. Thus we consider the normal form game where the agents' strategies are output demands and where cost shares are distributed according to a certain cost sharing mechanism. As is typical in settings 
like this involving increasing returns to scale, the resulting normal form game may not have any Nash equilibria. In fact, our first result is an impossibility theorem; we show that under increasing returns to scale, any smooth, and strictly monotone mechanism for which a Nash equilibrium exists for all profiles of convex and monotone preferences must be dictatorial. Therefore there can be no suitably well-behaved mechanism for which Nash equilibria always exist when preferences are only restricted to be convex and monotone.

The decreasing serial mechanism is not dictatorial. It follows as a corollary of the above result that this existence problem also applies to the game induced by the decreasing serial rule. But there is a domain restriction on preferences for which existence of a Nash equilibrium can be guaranteed. The subdomain of convex and monotone preferences that allows to guarantee existence of equilibrium for the decreasing serial game consists of the set of utility functions where the output is an inferior good. Quasi-linear utility functions, the utility functions most commonly used to represent preferences over cost shares, are in that subdomain. For these particular utility functions, the increasing serial mechanism ${ }^{1}$ also induces a cost sharing game that possesses at least one Nash equilibrium while the games induced by average cost pricing or marginal cost pricing fail to have any Nash equilibrium (see Moulin [7]).

Under this domain restriction the game induced by the decreasing serial rule is a game with strategic complementarities, that is to say, one for which each agent's best reply function is increasing in the other agents' strategies; hence, it always has a Nash equilibrium. It may have multiple Nash equilibria, but it has a largest and a smallest Nash equilibrium and all other equilibrium quantities lie between these bounds.

It turns out that the decreasing serial game has interesting welfare properties as well. If all agents have the same preferences, the equilibria of the decreasing serial game (if any exist) coincide with that from the increasing serial game and both mechanisms yield the first-best. This is not the case for the equilibria (if any exist) of the average cost game at which a uniform reduction of the common equilibria demands is Pareto improving. ${ }^{2}$ Other efficiency properties of the equilibria are the following. We

\footnotetext{
${ }^{1}$ Moulin [7] compares this mechanism against marginal cost pricing, average cost pricing and the Shapley-Shubik mechanism. These mechanisms are compared for the existence of Nash equilibrium demand profiles and for the equity properties of these outcomes. He shows that among the four only Moulin and Shenker mechanism and the Shapley-Shubik mechanism possess at least one Nash equilibrium on the domain of binormal preferences (both input and output are normal goods).

${ }^{2}$ Moulin and Watts prove a similar claim in [9]. They assume that cost is convex and preferences are binormal. They show that the only way to make agents better off than they are at the Nash equilibrium of the average cost sharing game is to decrease total production.
} 
show that if the serial game has a unique Nash equilibrium then the equilibrium must be strong. Thus no coalition can deviate without decreasing the utility of at least one of its members, assuming that the complementary coalition still uses the equilibrium strategy. If there exist multiple Nash equilibria, the smallest one (that is to say, the one in which each agent demands the least among her possible equilibrium strategies) is the unique equilibrium that is strong. Moreover, it is the Pareto superior equilibrium.

Finally, we show that the increasing and the decreasing serial rules are the only mechanisms with these strategic and welfare properties. This characterization theorem gives a strong argument in favor of serial cost sharing in the presence of economies of scale.

The remainder of the paper is outlined as follows. Section 2 provides the basic setup of the paper and it addresses the issue of existence of equilibrium in cost sharing games. In Section 3 we introduce the decreasing serial rule and state some of its normative properties. Section 4 is devoted to show the strategic and welfare properties of the noncooperative outcome of the game induced by the decreasing serial rule. This section contains a characterization theorem of the serial rules based on incentive properties. Finally, the Appendixes provide the proofs of our two main theorems and of some auxiliary results.

\section{COST SHARING GAMES}

\subsection{Basic Setup}

A group of agents $(N=\{1, \ldots, n\})$ share a production process that transforms a single (divisible) input into a single homogeneous (divisible) output. Each agent demands a certain amount of the produced output $\left(q_{i}, i=1, \ldots, n\right)$. The total cost of providing these demands is $C\left(\sum_{i=1}^{n} q_{i}\right)$. Throughout the paper, $C(\cdot)$ is assumed to be an increasing and strictly concave function, with $C(0)=0$. We denote by $\mathrm{C}$ the set of such functions.

In a cost sharing game, each agent simultaneously demands an amount of output; the cost of producing this output is then divided by a given cost sharing mechanism.

A cost sharing mechanism is a mapping $\xi$ associating to each cost function $C$, and vector of demands $\mathbf{q}=\left(q_{1}, \ldots, q_{n}\right)$ in $\mathscr{R}_{+}^{n}$, a vector of cost shares $\left(x_{1}, \ldots, x_{n}\right)$ such that

$$
\left(x_{1}, \ldots, x_{n}\right)=\left(\xi_{1}(C ; \mathbf{q}), \ldots, \xi_{n}(C ; \mathbf{q})\right) \quad \text { and } \quad \sum_{i=1}^{n} x_{i}=C\left(\sum_{i=1}^{n} q_{i}\right) .
$$

Each individual has preferences over the possible vectors input-output, $\left(q_{i}, x_{i}\right)$. Agent $i$ 's preferences are nondecreasing in her output share, $q_{i}$, 
nonincreasing in her input share, $x_{i}$, and they can be represented by a utility function $u_{i}\left(q_{i}, x_{i}\right)$.

Given a cost sharing mechanism $\xi$, a cost function $C$ in $\mathrm{C}$, and a preference profile $\left(u_{1}, \ldots, u_{n}\right)$, the corresponding cost sharing game $G(C$; $u_{1}, \ldots, u_{n}$ ) has the strategy set $\mathscr{R}_{+}$for each player and the payoff function $u_{i}\left(q_{i}, \xi_{i}\left(C ; q_{1}, \ldots, q_{n}\right)\right)$ for player $i$.

The following domain of preferences and endowments is maintained throughout.

$\mathscr{D}$ : Preferences can be represented by strictly quasiconcave $\mathscr{C}^{2}$ utility functions from $\mathscr{R}_{+}^{2}$ into $\mathscr{R}$ satisfying the following additional property:

(A1) Each individual endowment of input is uniformly bounded. That is: There is $M<\infty$ such that for all elements in the domain, $x_{i} \leqslant M$.

Assumption (A1) states that each agent has finite wealth. An alternative assumption, equivalent to (A1), would be to consider that indifference curves in the $\left(q_{i}, x_{i}\right)$ plane converge to zero as $q_{i} \rightarrow \infty$. This implies that for each agent there exists a level of output for which the extra payment that she is willing to make in order to get an additional unit of the good is smaller than the marginal cost of producing it, so that no agent will demand unbounded quantities of output.

We now turn to interesting subdomains of $\mathscr{D}$.

$\mathscr{D}_{\mathcal{N}}$ : Subdomain of $\mathscr{D}$ containing binormal preferences. Both goods are normal for an agent: For all $p_{1}, p_{2} \in \mathscr{R}_{+}$if $z=(q, x)$ is in the demand set on a given budget line $p_{1} q-p_{2} x \leqslant b$ and if $b^{\prime}>b$, then the demand set on the budget line $p_{1} q-p_{2} x \leqslant b^{\prime}$ contains at least a point $z^{\prime}=\left(q^{\prime}, x^{\prime}\right)$ such that $q^{\prime} \geqslant q, x^{\prime} \leqslant x$.

If preferences can be represented by a differentiable utility function, normality plus convexity is equivalent to the marginal rate of substitution-MRS, from now on-being nonincreasing in $x$ and $q$. Recall that the marginal rate of substitution is the slope $d x / d q$ of the indifference contour. The utility function $u(q, x)=a(q)-b(x)$ with $a(\cdot)$ a concave function and $b(\cdot)$ a convex function is an example of a utility function in this subdomain.

$\mathscr{D}_{\mathscr{\Phi}}:$ Subdomain of $\mathscr{D}$ containing preferences where $q$ is an inferior good. The output is an inferior good for an agent: For all $p_{1}, p_{2} \in \mathscr{R}_{+}$if $z=(q, x)$ is in the demand set on a given budget line $p_{1} q-p_{2} x \leqslant b$ and if $b^{\prime}>b$, then the demand set on the budget line $p_{1} q-p_{2} x \leqslant b^{\prime}$ contains at least a point $z^{\prime}=\left(q^{\prime}, x^{\prime}\right)$ such that $q^{\prime} \leqslant q, x^{\prime} \leqslant x$.

If preferences can be represented by a differentiable utility function this condition is equivalent to the MRS being nondecreasing in $x$. The utility 
function $u(q, x)=c(q)-d(x)$ with $c(\cdot)$ and $d(\cdot)$ concave functions is an example of a utility function in this subdomain.

\subsection{Nash Equilibrium}

Our objective is to study the noncooperative equilibria of cost sharing games. We begin by considering Nash equilibria. Nonexistence of Nash equilibria would pose a fundamental difficulty for the decentralized use of a mechanism.

Moulin [7] has conjectured that whenever the cost function is concave there is no anonymous mechanism guaranteeing at least one Nash equilibrium for all profiles of preferences in $\mathscr{D}$. This is the case for the normal form games induced by some of the best known mechanisms; those based on average or marginal cost pricing. Consider, for instance, the following example due to Moulin which illustrates the lack of Nash equilibrium for the game induced by the marginal cost pricing mechanism. Two consumers with utility functions $U_{1}\left(q_{1}, x_{1}\right)=\min \left\{q_{1}, 0.25 q_{1}+1.5\right\}-x_{1}$ and $U_{2}\left(q_{2}, x_{2}\right)=\min \left\{3 q_{2}, 0.2 q_{1}+19\right\}-x_{2}$ share a technology such that $C(q)=\min \{2 q, 0.5 q+15\}$. The nonexistence of Nash equilibrium at this preference profile is due to the discontinuity of the cost shares of agent 2 . It is easy to see that the best reply functions are:

$$
\begin{aligned}
& B R_{1}\left(q_{2}\right)=\left\{\begin{array}{lll}
0 & \text { if } & q_{2}<10 \\
2 & \text { if } & q_{2} \geqslant 10,
\end{array}\right. \\
& B R_{2}\left(q_{1}\right)=\left\{\begin{array}{lll}
10-q_{1} & \text { if } & q_{1} \leqslant 3 \\
7 & \text { if } & q_{1} \geqslant 3 .
\end{array}\right.
\end{aligned}
$$

For any cost sharing mechanism, for a given choice $q_{-i}$ of strategies by the agents other than $i$, the opportunity set $\left\{\left(q_{i}, x_{i}\right)\right.$ such that $\left.x_{i}=\xi_{i}\left(q_{i}, q_{-i}\right)\right\}$, where, hereafter, $q_{-i}$ refers to the vector $\left(q_{1}, \ldots, q_{i-1}, q_{i+1}, \ldots, q_{n}\right)$, is typically the graph of a concave function (with variable $q_{i}$ and value $x_{i}$ ). Therefore even convex preferences may attain a maximum at two or more distinct points. Since agents may not have single-peaked preferences on the different opportunity sets they may face, the best reply correspondences need not be convex-valued and the cost sharing game may fail to have a Nash equilibrium.

We now show that for a certain family of mechanisms Moulin's conjecture is true. This family, that we will denote by $\Omega$, is composed of anonymous cost sharing mechanisms satisfying the following two properties:

(i) Smoothness: $\xi$ is continuously differentiable in $\mathscr{R}_{+}^{n}$.

(ii) Strict monotonicity: $\left(\partial \xi_{i} / \partial q_{i}\right)(\mathbf{q})>0$ for all $\mathbf{q}$ in $\mathscr{R}_{+}^{n}$ and for all $i$. 
THEOREM 1. If the cost function is strictly concave, any smooth and strictly monotonic mechanism for which a Nash equilibrium exists for all preference profiles in $\mathscr{D}$ must be dictatorial.

Proof. See Appendix 2.

This impossibility result contrasts dramatically with the situation of decreasing returns studied by Moulin and Shenker $[5,6]$. In the convex case there are many anonymous mechanisms for which existence of a Nash equilibrium at all profiles of monotonic and convex preferences is guaranteed. In particular, the increasing serial cost sharing mechanism induces a dominance solvable game whose unique equilibrium is also robust to coalition deviations. ${ }^{3}$

Given Theorem 1, if the cost function is concave we cannot study the strategic properties of a mechanism unless we can guarantee the existence of Nash equilibria. Thus, to study the strategic properties one should first search for a domain of preferences that guarantee the existence of Nash equilibria. This is the approach we take in our analysis of the decreasing serial cost mechanism, which we shall now introduce.

\section{THE DECREASING SERIAL RULE}

Moulin and Shenker [5] have introduced the idea of serial cost sharing. They have proposed a mechanism which allocates a portion of the cost of total supply to each consumer in order of increasing demand. Here we propose a variation of their mechanism allocating costs to consumers in decreasing order of demands. Our allocation scheme means that the agent with the greatest demand gets the largest surplus. To be more explicit let us now explain how to obtain the different agents' payment.

The agent with the largest demand, assumed to be the $n$th without loss of generality, will be the one generating the biggest externality, and the one contributing most to the total surplus. We would not like the largest demander to be hurt by the presence of other users demanding less. That is the reason why we want the largest demander to pay the average cost of providing $n$ demands identical to her. Hence we fix agent $n$ 's payment to be $C\left(n q_{n}\right) / n$, where $q_{n}$ is her demand.

The agent with the next largest demand, say the $(n-1)$ th, will pay the average cost of providing $n-1$ demands identical to her plus the demand of the $n$th agent, but taking into account the quantity already paid by that

\footnotetext{
${ }^{3}$ The game induced by the average cost pricing mechanism also possesses Nash equilibria. In addition, if preferences are restricted to be in $\mathscr{Z}_{\mathscr{N}}$ then the equilibrium is unique (see Watts [13]).
} 
agent. If the other $(n-1)$ agents were demanding as much as her a surplus of $(n-1) C\left(q_{n-1}\right)-\left[C\left((n-1) q_{n-1}+q_{n}\right)-C\left(n q_{n}\right) / n\right]$ would be generated. An equal share of this surplus among them leads to a payment of

$$
\frac{C\left((n-1) q_{n-1}+q_{n}\right)-C\left(n q_{n}\right) / n}{n-1} .
$$

This is the share we propose for the $(n-1)$ th agent. All other agents' payments are derived from the same reasoning.

To give the explicit formula we may assume $q_{1} \leqslant q_{2} \leqslant \cdots \leqslant q_{n}$, and invoke anonymity in all the other cases. Thus we set,

$$
\begin{aligned}
x_{n}^{D}(\mathbf{q})= & \frac{C\left(n q_{n}\right)}{n} \\
x_{n-1}^{D}(\mathbf{q})= & \frac{C\left((n-1) q_{n-1}+q_{n}\right)-x_{n}^{D}(\mathbf{q})}{n-1} \\
x_{n-2}^{D}(\mathbf{q})= & \frac{C\left((n-2) q_{n-2}+q_{n-1}+q_{n}\right)-x_{n-1}^{D}(\mathbf{q})-x_{n}^{D}(\mathbf{q})}{n-2} \\
& \vdots \\
x_{i}^{D}(\mathbf{q})= & \frac{C\left(i q_{i}+q_{i+1}+\cdots+q_{n}\right)-x_{i+1}^{D}(\mathbf{q})-\cdots-x_{n}^{D}(\mathbf{q})}{i}
\end{aligned}
$$

where $x_{i}^{D}$ is the amount that agent $i$ with demand $q_{i}$ will pay under the decreasing serial cost rule when $q=\left(q_{1}, q_{2}, \ldots, q_{n}\right)$. The solution to this recursive system yields the closed form:

$$
x_{i}^{D}(\mathbf{q})=\frac{C\left(q^{i}\right)}{i}-\sum_{j=i+1}^{n} \frac{C\left(q^{j}\right)}{j(j-1)}
$$

with $q^{i}=i q_{i}+q_{i+1}+\cdots+q_{n}$.

We can see from the above formula that in this mechanism there exists a hierarchy in the decision making process (Satterthwaite and Sonnenschein [11]); that an agent cannot affect the choices of the higher ranking agents although she will be affected by their choices. Since this hierarchy goes from the largest to the smallest demander we call this mechanism the decreasing serial mechanism. In Moulin and Shenker's increasing serial mechanism an agent's cost share is independent of demands higher than her own. Formally,

$$
x_{i}^{I}(\mathbf{q})=\frac{C\left(\hat{q}^{i}\right)}{n-i+1}-\sum_{j=1}^{i-1} \frac{C\left(\hat{q}^{j}\right)}{(n-j+1)(n-j)}
$$

with $\hat{q}^{i}=q_{1}+q_{2}+\cdots+(n+1-i) q_{i}$. 
Under constant marginal cost both mechanisms prescribe the same payments which coincide with those derived from the nucleolus of the cooperative game with characteristic function $v(S)=\sum_{i \in S} C\left(q_{i}\right)-C\left(\sum_{i \in S} q_{i}\right)$.

Let me now list some of the properties ${ }^{4}$ of the decreasing serial mechanism. We have divided these properties into two groups: Those that hold for any increasing $C$ with $C(0)=0$ and those that hold only for $C$ in C, i.e., properties that require the cost function to be concave as well.

1. For any increasing $C$ with $C(0)=0$ the decreasing serial mechanism satisfies:

Monotonicity. $x_{i}^{\boldsymbol{D}}(\mathbf{q})$ is nondecreasing in $q_{i}$. Monotonicity implies that if $q_{i} \leqslant q_{i}^{\prime}$ then $x_{i}^{D}\left(q_{i}, q_{-i}\right) \leqslant x_{i}^{D}\left(q_{i}^{\prime}, q_{-i}\right)$.

FAIR RANkING. If $q_{i} \leqslant q_{j}$ then $x_{i}^{D}(\mathbf{q}) \leqslant x_{j}^{D}(\mathbf{q})$.

SMoothness. If $C$ is continuously differentiable on $\mathscr{R}_{+}^{n}$, then $x_{i}^{D}(\mathbf{q})$ is continuously differentiable on $\mathscr{R}_{+}^{n}$ as a function of $\mathbf{q}$.

2. For any $C$ in $C$ the decreasing serial mechanism satisfies:

Cross Monotonicity. $x_{i}^{D}(\mathbf{q})$ is nondecreasing in $q_{j}$. This property implies that if $q_{j} \leqslant q_{j}^{\prime}$ then $x_{i}^{D}\left(q_{j}, q_{-j}\right) \leqslant x_{i}^{D}\left(q_{j}^{\prime}, q_{-j}\right)$.

Cross monotonicity is crucial for obtaining a lower bound on the amount any agent will pay under the decreasing serial mechanism. This bound, called the Unanimity Cost Bound, will play a central role in the strategic analysis of this mechanism.

UNANIMITY Cost Bound. ${ }^{5} x_{i}^{D}(\mathbf{q}) \geqslant C\left(n q_{i}\right) / n$ for all $\mathbf{q}$ and all $i$.

The Unanimity Cost Bound means that an agent must pay at least her fair share in the hypothetical situation where all individual demands coincide with her. Moulin [8] (see also [7]) has proposed this bound as a normative consequence of the fact that differences in individual demands should not bear differently on different agents. To see the relevance of this bound, consider for instance the average cost pricing which fails this bound. Let the cost function be $C(q)=\sqrt{q}$. Let us assume three agents demand respectively $q_{1}=3, q_{2}=9$ and $q_{3}=18$ units of output. The average

\footnotetext{
${ }^{4}$ Some of the proofs are quite trivial and hence they are omitted. The proof of the cross monotonicity of the decreasing serial rule is in Appendix 1.

${ }^{5}$ Notice that the Unanimity Cost Bound serves as a lower bound when the cost function is concave and as un upper bound when it is convex.
} 
cost pricing proposes payments of $0.54,1.62$, and 3.24 units, respectively, since with this mechanism agent $i$ will pay

$$
x_{i}^{A}(\mathbf{q})=\frac{q_{i}}{\sum_{j=1}^{n} q_{j}} \cdot C\left(\sum_{j=1}^{n} q_{j}\right) .
$$

One can argue that agents 1 and 2 are paying too little. They pay less than their Unanimity Cost Bound, resulting in an unfair charge to agent 3. If agent 3 were alone she would pay 4.24 units. Thus, the presence of other users saves her 1 unit. This agent, who is the one generating the biggest externality, has the smallest savings, since the first agent saves 1.19 units (1.73-0.54) and the second one 1.38 units (3-1.62).

From a normative viewpoint the main drawback of the mechanism that we propose is that it may fail the Stand-Alone Test. This test postulates that an agent should not pay more than what she would pay if she were the sole user of the technology.

The decreasing serial mechanism can violate this principle when there exists a big gap among the demands of the different users. Consider for instance that there are only two agents and assume that the first agent demands 50 units and the second demands 3 units. If the cost function is $C(q)=\sqrt{q}$ then the decreasing serial mechanism proposes cost shares of 5 units for the first agent and of 2.28 units for the second one, while if this second agent were alone, she would only pay 1.73 . Clearly, the more agents that are involved in the cost sharing problem the more likely it is that the mechanism will pass this test. In the example above if there is a third agent with a demand of 10 units then the decreasing serial rule will propose cost shares of 4.08, 2.14, and 1.71 units. All the agents are now paying less than the payments they would have made if they were alone. The result holds for any demand by the third agent in the interval $[3,14.8]$. In the same vein, if the demands are similar then the stand alone test is always passed. In the above example if the demand of the second agent is 7 units instead of 3 then the cost shares will be 5 units for the agent demanding 50 units and 2.54 for the agent demanding 7 units, while the payments if they were alone would be 7.07 for the first agent and 2.64 for the second.

If the cost function is concave the failure of the stand-alone test is bothersome. The argument for sharing the technology comes from the increasing returns which indicates that agents should naturally want to cost share. However, the incentive to get together and share costs is undermined if the cost sharing method gives some individuals an incentive to go off on their own. We would recommend the application of the decreasing serial rule only in those situations where this test is either irrelevant or it is always passed. It is irrelevant if the agents cannot produce the good by themselves (examples are the share of the cost of setting electricity lines or 
a water supply system) ${ }^{6}$ It is always passed if the demands are similar (an example can be sharing the costs of a gardener's service among the neighbors of a housing estate).

\section{PROPERTIES OF THE DECREASING SERIAL RULE}

In this section we study the strategic and the welfare properties of the decreasing serial rule. Among the strategic properties we provide results on existence and uniqueness of a noncooperative equilibrium for the decreasing serial game. We also provide a welfare analysis of the rule. In particular, we provide results about the robustness of the equilibrium with respect to coalitional deviations as understood in the definition of the strong equilibrium). Finally, we prove that the serial mechanisms (increasing or decreasing) are the only methods with such appealing strategic and welfare properties.

We begin by introducing some notation.

We will denote a vector in $\mathscr{R}_{+}^{n}$ by $\mathbf{q}$ or by $\left(q_{i}\right)_{i \in N}$. We write $\left(i \cdot q, q_{i+1}, \ldots, q_{n}\right)$ for the vector in $\mathscr{R}_{+}^{n}$ with the first $i$ components equal to $q$. Pick any ordered vector q. For $i=1, \ldots, n-1$ define

$$
\eta\left(i ; q_{i+1}, \ldots, q_{n}\right)=\left\{(q, x) \text { such that } x= \begin{cases}x_{n}^{D}(n \cdot q) & \text { if } q \geqslant q_{n} \\ x_{n-1}^{D}\left((n-1) \cdot q, q_{n}\right) & \text { if } q_{n-1} \leqslant q \leqslant q_{n} \\ \vdots & \\ x_{i}^{D}\left(i \cdot q, q_{i+1}, \ldots, q_{n}\right) & \text { if } q \leqslant q_{i+1} .\end{cases}\right.
$$

For $i=n$ write $\eta(n)$ for the set of allocations $(q, x)$ such that $x=C(n q) / n$. Fix the demands of all agents $j, j \neq k$, and order them such that $q_{1} \leqslant \cdots \leqslant q_{n-1}$. Then agent $k$ 's opportunity set is the set of allocations she can reach by demanding any quantity of the good. We will denote this opportunity set by $\Phi\left(k ; q_{-k}\right)$. If the cost function is smooth then the decreasing serial mechanism is also smooth as a function of $\mathbf{q}$, and every agent's opportunity set will be a smooth curve as well. Indeed, it will be a continuous and concave curve (with variable $q_{k}$ and value $x_{k}$ ) that consists of $n$ pieces. Its graph is $\Phi\left(k ; q_{-k}\right)=\eta\left(1 ; q_{1}, \ldots, q_{n-1}\right)$.

\footnotetext{
${ }^{6}$ In many situations agents cannot produce the good by themselves due to governmental regulations. Think of the Electric Power Sector. In the United Kingdom and Spain, for example, generators and distribution centers are constrained by a fixed transmission system. Generator companies might be better off having their own transmission system but the government does not allow them to do so. Also, consider the case of petroleum refining in Uruguay. It is illegal to construct a refinery. However the law mandates that all petroleum exporter companies use the state-owned refinery ANCAP.
} 
Let us illustrate the definition of opportunity set with an example. Let $C(q)=\sqrt{q}$ and let $n=3$. Let us name the agents $a, b$, and $c$ and let $q_{a}=20$, and $q_{b}=10$. For this example agent $c$ 's opportunity set will be:

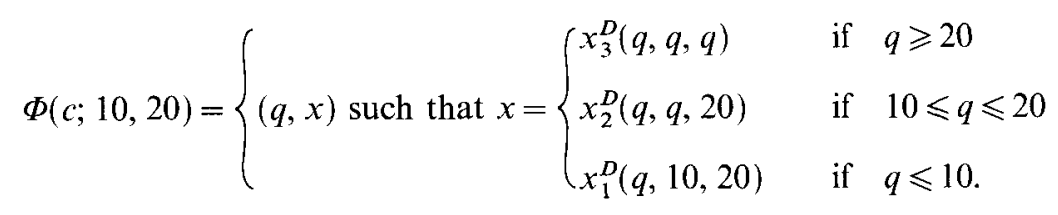

We define an agent's optimistic utility as the maximum utility level an agent can reach on the different opportunity sets she faces. In particular, agent $i$ 's, $i=1, \ldots, n$, optimistic utility on the set $\eta(n)$ will be the following utility level:

$$
\begin{aligned}
& \max _{q_{i}} u_{i}\left(q_{i}, x_{i}\right) \\
& \text { s.t. } q_{i} \geqslant 0 \\
& x_{i}=C\left(n q_{i}\right) / n .
\end{aligned}
$$

Agent $i$ 's optimistic demand on $\eta(n)$ is the set of solutions to the above program. A solution exists since the utility function is continuous and nonincreasing in the payment and the constrained set is nonempty and compact (see (A1)).

The optimistic demands will play a central role in the study of the strategic properties of the decreasing serial game. They are crucial to compute the Nash equilibrium of this game. On the one hand, the multiplicity of optimistic demands may yield to best reply correspondences that are not convex-valued and to the nonexistence of equilibrium. But, on the other hand, the uniqueness of the optimistic demands yields uniqueness of equilibrium. Theorem 3 in this section hinges on and shows these results.

\subsection{Strategic Properties}

\subsubsection{Existence of Nash Equilibrium}

Theorem 1 tells us that there can be no suitably well-behaved mechanism for which Nash equilibria always exist when preferences are only restricted to be convex and monotone. Since this result applies to the decreasing serial mechanism, one could deal with the existence problem by looking for domain restrictions on preferences. For any possible domain restriction we cannot use the fixed points theorems from convex analysis because the best 
reply correspondences are not typically convex-valued. Nevertheless, we now show that there exists a subdomain of $\mathscr{D}, \mathscr{D}_{\mathscr{I}}$, for which the fixed point theorems from lattice theory can be fruitfully applied to the decreasing serial game. Thus we provide a subdomain of $\mathscr{D}$ for which existence can be guaranteed. The kind of utility functions contained in $\mathscr{D}_{\mathscr{S}}$ include quasilinear utility functions $(v(q)-x$, with $v$ concave and increasing) or CES utility functions $\left(q^{p}-x^{p}, 0<p<1\right)$.

If the preference profiles are in $\mathscr{D}_{\mathscr{S}}$ then the decreasing serial game is a game with strategic complementarities. The simplicity of these games makes convexity and differentiability assumptions unnecessary. What is needed is an order structure on strategy spaces, a weak continuity requirement on payments, and the property that players' marginal utility be monotone in the strategies of their rivals.

The formal definitions ${ }^{7}$ are as follows.

Definition 1. A game with strategic complementarities is such that for each player $i$ :

(i) The strategy set $S_{i}$ is a compact lattice;

(ii) The payoff function $u_{i}$ is upper semicontinuous in $s_{i}$ for $s_{-i}$ fixed, and continuous in $s_{-i}$ for fixed $s_{i}$;

(iii) $u_{i}$ is quasi-supermodular in $s_{i}$ and satisfies the single crossing property in $\left(s_{i} ; s_{-i}\right)$.

Definition 2. $u_{i}\left(s_{i}, s_{-i}\right)$ satisfies the single crossing property in $\left(s_{i} ; s_{-i}\right)$, if for all $s_{i}, \tilde{s}_{i} \in S_{i}$, and $s_{-i}, \tilde{s}_{-i} \in S_{-i}$ such that $s_{i}>\tilde{s}_{i}$ and $s_{-i}>\tilde{s}_{-i}$ one has that

$$
\begin{aligned}
& u_{i}\left(s_{i}, \tilde{s}_{-i}\right)>u_{i}\left(\tilde{s}_{i}, \tilde{s}_{-i}\right) \text { implies } u_{i}\left(s_{i}, s_{-i}\right)>u_{i}\left(\tilde{s}_{i}, s_{-i}\right), \text { and } \\
& u_{i}\left(s_{i}, \tilde{s}_{-i}\right) \geqslant u_{i}\left(\tilde{s}_{i}, \tilde{s}_{-i}\right) \text { implies } u_{i}\left(s_{i}, s_{-i}\right) \geqslant u_{i}\left(\tilde{s}_{i}, s_{-i}\right) .
\end{aligned}
$$

DeFINITION 3. $u_{i}\left(s_{i}, s_{-i}\right)$ is quasi-supermodular in $s_{i}$ if for each $s_{-i}$ and for all $s_{i}, \tilde{s}_{i} \in S_{i}$, and,

(i) $u_{i}\left(s_{i}, s_{-i}\right) \geqslant u_{i}\left(s_{i} \wedge \tilde{s}_{i}, s_{-i}\right)$ implies $u_{i}\left(s_{i} \vee \tilde{s}_{i}, s_{-i}\right) \geqslant u_{i}\left(\tilde{s}_{i}, s_{-i}\right)$,

(ii) $u_{i}\left(s_{i}, s_{-i}\right)>u_{i}\left(s_{i} \wedge \tilde{s}_{i}, s_{-i}\right)$ implies $u_{i}\left(s_{i} \vee \tilde{s}_{i}, s_{-i}\right)>u_{i}\left(\tilde{s}_{i}, s_{-i}\right)$, where $s_{i} \wedge \tilde{s}_{i}$ denote the greatest lower bound, or meet of $s_{i}$ and $\tilde{s}_{i}$ and $s_{i} \vee \tilde{s}_{i}$ denote the least upper bound, or join of $s_{i}$ and $\tilde{s}_{i}$.

${ }^{7}$ All the definitions that follow have been taken from Milgrom and Shannon [4]. 
The games with strategic complementarities have a series of useful properties, including existence of pure strategy equilibria, monotone comparative statics on equilibrium sets, coincidence of the predictions of various solutions concepts, stability under adaptive learning, and certain welfare properties of the equilibria. In the theorems that follow we show that the game induced by the decreasing serial mechanism has a nonempty equilibrium set in which a largest and a smallest Nash equilibrium exist (Theorem 2 below). Its largest Nash equilibrium is the largest strategy surviving the iterative elimination of strictly dominated strategies. In the welfare analysis of the decreasing serial rule we also show that the smallest Nash equilibrium is the only equilibrium that is a strong equilibrium; moreover, it is the Pareto superior among all the equilibria (these results are established in Proposition 1).

THEOREM 2. For all $C \in \mathrm{C}$ and all $\left(u_{1}, \ldots, u_{n}\right)$ in $\mathscr{D}_{\mathfrak{g}}$ the decreasing serial game is a game with strategic complementarities. Its set of pure strategy Nash equilibria is nonempty and possesses greatest and least equilibrium points.

Proof. The decreasing serial game has quasi-supermodular payoff functions by the single-dimensionality of the strategy sets. Its payoff functions are continuous due to the continuity of the decreasing serial rule and of the utility functions. The strategy set for each player $i, S_{i}=\left[0, \tilde{q}_{i}\right]$, is a compact lattice because of (A1). Recall that (A1) guarantees that no agent will demand unbounded quantities of output. Thus, to prove the statement of the theorem, we have only to show that the payoff functions satisfy the single crossing property.

Let us denote $x_{i}^{D}\left(q_{i}^{\prime}, q_{-i}^{\prime}\right)$ by $y^{\prime}, x_{i}^{D}\left(q_{i}^{\prime}, q_{-i}\right)$ by $x^{\prime}, x_{i}^{D}\left(q_{i}, q_{-i}\right)$ by $x$, and finally, $x_{i}^{D}\left(q_{i}, q_{-i}^{\prime}\right)$ by $y$. We have to show that for all $q_{-i}^{\prime}>q_{-i}$ and $q_{i}^{\prime}>q_{i}$, if $u_{i}\left(q_{i}^{\prime}, x^{\prime}\right) \geqslant u_{i}\left(q_{i}, x\right)$ then $u_{i}\left(q_{i}^{\prime}, y^{\prime}\right) \geqslant u_{i}\left(q_{i}, y\right)$; and if $u_{i}\left(q_{i}^{\prime}, x^{\prime}\right)>u_{i}\left(q_{i}, x\right)$ then $u_{i}\left(q_{i}^{\prime}, y^{\prime}\right)>u_{i}\left(q_{i}, y\right)$. To prove it we first show some results on the assignment of cost shares by the decreasing serial rule.

The monotonicity of the decreasing serial rule ensures that $y^{\prime}>y$ and $x^{\prime}>x$, and the cross monotonicity property ensures that $y^{\prime}>x^{\prime}$. Finally, since the cost function is concave, we have $x^{\prime}-x \geqslant y^{\prime}-y$; note that

$$
x^{\prime}-x=C\left(i q_{i}^{\prime}+\sum_{j>i} q_{j}\right)-C\left(i q_{i}+\sum_{j>i} q_{j}\right),
$$

and

$$
y^{\prime}-y=C\left(i q_{i}^{\prime}+\sum_{j>i} q_{j}^{\prime}\right)-C\left(i q_{i}+\sum_{j>i} q_{j}^{\prime}\right) .
$$


Consider four possible cost shares for agent $i$, let these be $\alpha^{\prime}, \alpha, \beta^{\prime}$ and $\beta$, with $\alpha^{\prime}>\alpha, \beta^{\prime}>\beta$ and $\beta^{\prime}>\alpha^{\prime}$. Because the utility functions are in $\mathscr{D}_{\mathscr{S}}$ we know that if $q_{i}^{\prime}>q_{i}$ and if $u_{i}\left(q_{i}, \alpha\right)=u_{i}\left(q_{i}^{\prime}, \alpha^{\prime}\right)$ and $u_{i}\left(q_{i}, \beta\right)=u_{i}\left(q_{i}^{\prime}, \beta^{\prime}\right)$ hold, then $\beta^{\prime}-\beta \geqslant \alpha^{\prime}-\alpha$.

Assume first $u_{i}\left(q_{i}^{\prime}, x^{\prime}\right) \geqslant u_{i}\left(q_{i}, x\right)$. The continuity of the preferences implies that there exists $z$, with $z \leqslant x$, for which $u_{i}\left(q_{i}^{\prime}, x^{\prime}\right)=u_{i}\left(q_{i}, z\right)$, and that there exists $t, t \geqslant z$, for which $u_{i}\left(q_{i}^{\prime}, y^{\prime}\right)=u_{i}\left(q_{i}, t\right)$. From the argument above, and since the utility functions are in $\mathscr{D}_{\mathscr{I}}$, it follows that $y^{\prime}-t \geqslant x^{\prime}-z$.

We now show that $t$ must be smaller than or equal to $y$. Assume the opposite, that is, consider $t>y$. We get $y^{\prime}-y>y^{\prime}-t \geqslant x^{\prime}-z \geqslant x^{\prime}-x$, but this contradicts the concavity of the cost function. Hence $t$ cannot be bigger than $y$, and thus, since the utility functions are nonincreasing in the payment, we can conclude that $u_{i}\left(q_{i}^{\prime}, y^{\prime}\right)=u_{i}\left(q_{i}, t\right) \geqslant u_{i}\left(q_{i}^{\prime}, y\right)$. Thus, for all $q_{-i}^{\prime}>q_{-i}$ and $q_{i}^{\prime}>q_{i}$, if $u_{i}\left(q_{i}^{\prime}, x^{\prime}\right) \geqslant u_{i}\left(q_{i}, x\right)$ then $u_{i}\left(q_{i}^{\prime}, y^{\prime}\right) \geqslant u_{i}\left(q_{i}, y\right)$.

Assume now $u_{i}\left(q_{i}^{\prime}, x^{\prime}\right)>u_{i}\left(q_{i}, x\right)$. Because of continuity of the preferences we know that there exist $z$ and $t$ with $t>z, x>z$, for which $u_{i}\left(q_{i}^{\prime}, x^{\prime}\right)=u_{i}\left(q_{i}, z\right)$ and $u_{i}\left(q_{i}^{\prime}, y^{\prime}\right)=u_{i}\left(q_{i}, t\right)$. We have to show that $t$ is smaller than $y$. Because the utility functions are in $\mathscr{D}_{\mathscr{F}}$, it follows that $y^{\prime}-t \geqslant x^{\prime}-z$, whereas the concavity of the cost function implies $x^{\prime}-x \geqslant y^{\prime}-y$. Hence we have $y^{\prime}-t \geqslant x^{\prime}-z>x^{\prime}-x \geqslant y^{\prime}-y$, and therefore $y>t$.

We have just shown that the decreasing serial game satisfies the single crossing property, thus we can ensure that the decreasing serial game belongs to the class of games with strategic complementarities. This implies that its equilibrium set is nonempty and a largest ${ }^{8}$ and a smallest Nash equilibrium exist (see Vives [12]).

According to the above theorem the decreasing serial game never fails to have a Nash equilibrium when preferences are represented by quasilinear utility functions. These utility functions constitute the foremost subdomain to check for existence of equilibrium in any cost sharing game. From Moulin [7] we know that the increasing serial game always has at least one Nash equilibrium when preferences are in $\mathscr{D}_{N}$ (therefore, in particular, for quasilinear utility functions). This is not the case for the average cost pricing game or the marginal cost pricing game that may not have any Nash equilibrium when agents have quasilinear utility functions. Moulin's result and Theorem 2 together create a strong argument in favor of serial cost sharing (decreasing and/or increasing) in the presence of economies of scale.

\footnotetext{
${ }^{8}$ The largest Nash equilibrium is the largest strategy surviving the iterative elimination of strictly dominated strategies (see Theorem 10 in Milgrom and Roberts [3]).
} 


\subsubsection{Uniqueness of Equilibrium}

Results on existence of equilibrium for the whole family of convex and monotone preferences ${ }^{9}$ are required to rely on joint conditions on the preferences and on the cost function. We have found a sufficient condition on curvature of preferences and payment schedules which guarantees the existence of a (unique) Nash equilibrium for the entire domain of convex and monotone preferences. It requires indifference curves to have a bigger gaussian curvature than that of the mechanism. ${ }^{10}$ Results involving curvature restrictions are standard means of getting existence results in the presence of nonconvexities. Our sufficient condition ${ }^{11}$ allows us to prove not only existence but also uniqueness of Nash equilibrium. Uniqueness is a requirement to predict the outcome of a game and it is important for comparative statics analysis.

Hence we now consider the domain of convex and monotone preferences satisfying the following condition:

(AU) Given $C \in \mathrm{C},\left|\left(d / d q_{i}\right)\left[M R S^{i}\left(q_{i}, x_{i}^{D}(\mathbf{q})\right)\right]\right|>\left|\left(d^{2} / d q_{i}^{2}\right) x_{i}^{D}(C ; \mathbf{q})\right|$ for all $\mathbf{q} \in \mathscr{R}_{+}^{n}$ and all $\left(q_{i}, x_{i}^{D}(\mathbf{q})\right)$.

Assumption (AU) is a condition that is sufficient enough to ensure that each agent has single-peaked preferences on any opportunity set ${ }^{12}$ that she may face given the decreasing serial rule. In the situation where all agents have the same preferences (AU) is equivalent to ensuring that agents have single-peaked preferences on the opportunity set $C(n q) / n$. The next lemma, whose proof is in Appendix 1, shows this result.

\section{Lemma 1. Given $C \in \mathrm{C}$ and $\left(u_{1}, \ldots, u_{n}\right)$ in $\mathscr{D}$ if $(A U)$ holds relative to $C$ then:}

(i) Each agent has a unique optimistic demand on any opportunity set given for the decreasing serial rule.

\footnotetext{
${ }^{9}$ Consider, for instance, the game in which two agents, let us say $A$ and $B$, with preferences $u_{A}=\sqrt{40 q_{A}}-x_{A}^{2}$ and $u_{B}=3 \sqrt{q_{B}}-x_{B}^{2}$ have to decide their optimal demand. Assume the production cost is $C(q)=\sqrt{q}$. These preferences are not in $D_{\mathscr{f}}$ but equilibrium exists; moreover, it is unique. It corresponds to the strategies $\left(q_{A}=40, q_{B}=14.5\right)$. Notice that this does not imply that this subdomain is not tight, it just shows that by imposing a joint condition on the domain of preferences and the cost function, it may be possible to have some other existence results. We show that this is indeed the case.

${ }^{10} \mathrm{~A}$ constraint based in gaussian curvatures is used by Balasko and Shell [1] to show the optimality of the monetary equilibrium in a model of overlapping generations.

${ }^{11}$ It is a similar condition to the one proposed by Quinzii [10] to show the nonemptyness of the core of a economy with increasing returns to scale.

${ }^{12}$ If the utility functions are homothetic (Cobb-Douglas, for example), or the cost function is such that the marginal cost is concave, a weaker condition may be imposed. Single-peakedness over the set $C(n q) / n$ will be enough to ensure single-peakedness over any other possible opportunity set.
} 
(ii) Fix $q_{i+1}, \ldots, q_{n}$ such that $q_{i+1} \leqslant \ldots \leqslant q_{n}$. For every $j, j=1, \ldots, i$, let $q_{j}^{\prime}$ be agent $j$ 's optimistic demand on the set $\eta\left(k ; q_{i+1}, \ldots, q_{n}\right)$. If $q_{i}^{\prime} \geqslant q_{j}^{\prime}$ for all $j \neq i$, then agent $j$ 's optimistic demand on the set $\eta\left(k-1 ; q_{i}^{\prime}, q_{i+1}, \ldots, q_{n}\right)$ will never be greater than $q_{i}^{\prime}$.

If Condition (AU) holds relative to $C$ then the cost sharing game $G\left(C ; u_{1}, \ldots, u_{n}\right)$ induced by the decreasing serial mechanism is dominance solvable, therefore it has a unique equilibrium. ${ }^{13}$

THEOREM 3. For $C \in \mathrm{C}$ given and for any $\left(u_{1}, \ldots, u_{n}\right)$ in $\mathscr{D}$, if Condition $(A U)$ holds relative to $C$, then the decreasing serial game has a unique Nash equilibrium.

Proof. The proof of the theorem goes as follows: we first define via induction a certain outcome $\left(q_{i}^{*}, x_{i}^{*}\right)_{i \in N}$ and then we show that $\left(q_{i}^{*}\right)_{i \in N}$ is the unique Nash equilibrium of the decreasing serial game.

In order to define $\left(q_{i}^{*}, x_{i}^{*}\right)_{i \in N}$, we start by solving for each agent $i$, $i=1, \ldots, n$, the following program:

$$
\begin{aligned}
& \max _{q_{i}} u_{i}\left(q_{i}, x_{i}\right) \\
& \text { s.t. } q_{i} \geqslant 0 \\
& x_{i}=\frac{C\left(n q_{i}\right)}{n} .
\end{aligned}
$$

Let $\tilde{q}_{i}$ be the solution to the above program. Assumption (A1) on preferences ensures that a solution exists and part (i) of Lemma 1 ensures that it is unique. Pick the agent with the largest $\tilde{q}_{i}$, let this be the $n$th agent. In any Nash equilibrium agent $n$ 's strategy must be $\tilde{q}_{n}$, since this agent attains her maximum possible utility level whenever she uses $\tilde{q}_{n}$. So we set $q_{n}^{*}=\tilde{q}_{n}$ and $x_{n}^{*}=C\left(n \tilde{q}_{n}\right) / n$.

Next we solve for each agent $i, i<n$, the program:

$$
\max _{q_{i}} u_{i}\left(q_{i}, x_{i}\right)
$$

$$
\begin{array}{ll}
\text { s.t. } & q_{i} \geqslant 0 \\
& x_{i}=\frac{C\left((n-1) q_{i}+q_{n}^{*}\right)-x_{n}^{*}}{n-1} .
\end{array}
$$

${ }^{13}$ One could also define a condition similar to (AU) for the increasing serial rule and have an analogous result. 
We denote by $\hat{q}_{i}$ the unique solution to this program. We pick the agent with the largest $\hat{q}_{i}$, without loss of generality let this be the $(n-1)$ th agent. Part (ii) of Lemma 1 implies that $\hat{q}_{n-1}<\tilde{q}_{n}$, since $\tilde{q}_{n-1}$ was smaller than $\tilde{q}_{n}$. Hence $q_{n-1}^{*}=\hat{q}_{n-1}$ and $x_{n-1}^{*}=\left(C\left((n-1) q_{n-1}^{*}+q_{n}^{*}\right)-x_{n}^{*}\right) /(n-1)$.

Assume we have constructed the non-increasing sequence $\left(q_{i}^{*}, x_{i}^{*}\right)$ for $i=k, k+1, \ldots, n$. For each agent $s, s<k$, we compute her optimistic demand on the set $\eta\left(k ; q_{i}^{*}, \ldots, q_{n}^{*}\right)$. Let us denote these optimistic demands $\bar{q}_{1}, \ldots, \bar{q}_{k-1}$. Assume agent $(k-1)$ th's optimistic demand is the largest one, then we set $q_{k-1}^{*}=\bar{q}_{k-1}$ and $x_{k-1}^{*}=x_{k-1}^{D}\left((k-1) \cdot q_{k-1}^{*}, q_{k}^{*}, \ldots, q_{n}^{*}\right)$. The definition is then completed by induction.

We now show that $\left(q_{1}^{*}, \ldots, q_{n}^{*}\right)$ is a Nash equilibrium of the decreasing serial game, i.e., we prove that for every $i \in N, q_{i}^{*}=\arg \max _{q_{i}} u_{i}\left(q_{i}, x_{i}^{D}\left(q_{i}, q_{-i}^{*}\right)\right)$.

Pick an agent $i, i \in N$. Since $q_{i}^{*}$ is, by definition, agent $i$ 's optimistic demand on the set $\eta\left(k ; q_{i+1}^{*}, \ldots, q_{n}^{*}\right)$, we know that $q_{i}^{*}=\arg \max _{q_{i}} u_{i}\left(q_{i}\right.$, $\left.x_{i}^{D}\left(i \cdot q_{i}^{*}, q_{i+1}^{*}, \ldots, q_{n}^{*}\right)\right)$. Now (ii) of Lemma 1 implies that $q_{i}^{*}$ is agent $i$ 's optimistic demand on the opportunity set $\Phi\left(i ; q_{-i}^{*}\right)$, thus $q_{i}^{*}=$ $\arg \max _{q_{i}} u_{i}\left(q_{i}, x_{i}^{\boldsymbol{D}}\left(q_{i}, q_{-i}^{*}\right)\right)$; and this holds for all $i, i=1, \ldots, n$.

In any Nash equilibrium each player's strategy must be an optimal response to the other players' strategies. Since the sequence $\left(q_{i}^{*}\right)_{i \in N}$ contains the players' optimistic demands on the different opportunity sets they face given the other players' demands, it is the sequence of optimal responses. The uniqueness of the sequence of optimal responses ensures the uniqueness of the Nash equilibrium of the decreasing serial game.

The proof of the above theorem provides an algorithm to compute the Nash equilibrium of the decreasing serial game, whenever (AU) holds. We first compute the optimistic demands of all the agents on the opportunity set $C(n q) / n$. Pick the agent with the largest one and denote this agent $n$. Agent $n$ 's optimistic demand on the set $C(n q) / n$, let this be $q_{n}^{*}$, is her Nash equilibrium strategy. We then compute the optimistic demands of the remainder $(n-1)$ agents on the set $\eta\left(n-1 ; q_{n}^{*}\right)$. Pick the agent with the largest one and denote this agent $n-1$. The largest optimistic demand on the set $\eta\left(n-1 ; q_{n}^{*}\right)$ will be the Nash equilibrium strategy of agent $n-1$, and so on. From the proof it is easy to see that the decreasing serial game is dominance solvable: the successive elimination of strictly dominated strategies converges to the unique Nash equilibrium of the game. Obviously, the first round of deletion of strictly dominated strategies results in the strategy sets being restricted to those strategies not bigger than $q_{n}^{*}$.

\subsubsection{Welfare Properties}

We have just shown two results on existence of a noncooperative equilibrium for the decreasing serial game. 
Theorem 2 tells us that if preferences are in $\mathscr{D}_{\mathscr{I}}$ the decreasing serial rule induces a game for which a Nash equilibrium always exists. The result in Theorem 2 does not preclude the multiplicity of equilibria at some preferences profiles in $\mathscr{D}_{\mathscr{A}}$. Despite this multiplicity Nash equilibrium behavior in the decreasing serial game has a Pareto superior selection which is the smallest Nash equilibrium. It is also a strong equilibrium. If communication between players is allowed it is predictable that players will agree on this particular equilibrium which determines the outcome of the game.

Theorem 3 states that condition (AU) is sufficient to ensure the uniqueness of a noncooperative equilibrium for the decreasing serial game at all profiles of convex and monotone preferences. This unique equilibrium is a strong equilibrium as well. When all agents have the same preferences, condition ( $\mathrm{AU}$ ) ensures the existence and uniqueness of an equilibrium for either serial game (increasing and/or decreasing). This unique equilibrium gives the agents a first best outcome. This is not the case for the average cost pricing game where the equilibrium, if it exists, is not first best efficient.

These interesting welfare properties of the decreasing serial game are summarized in the following propositions.

Proposition 1. For all $C \in \mathrm{C}$ and all $\left(u_{1}, \ldots, u_{n}\right)$ in $\mathscr{D}_{\mathscr{G}}$ the smallest Nash equilibrium of the decreasing serial cost sharing game is the unique strong equilibrium of the game: no coalition can deviate without decreasing the utility of at least one of its members, assuming that the complementary coalition still uses the equilibrium strategy. Moreover it is the Pareto superior Nash equilibrium while the largest Nash equilibrium is the Pareto inferior Nash equilibrium.

Proof. Let us assume $\left(q_{i}\right)_{i \in N}$ is the smallest Nash equilibrium of the decreasing serial game. Suppose coalition $S$ deviates from this equilibrium by using strategies $\left(\tilde{q}_{i}\right)_{i \in S}$. Let $Y=\left\{i \in S\right.$ such that $\left.q_{i} \neq \tilde{q}_{i}\right\}$ and let $\mathbf{z}, \mathbf{z} \in \mathscr{R}^{\#}$, be the vector defined by $z_{i}=\max \left\{q_{i}, \tilde{q}_{i}\right\}$ with $i \in Y$. Let $k$ be the maximum element in z, i.e., $z_{k} \geqslant z_{j}$ for all $j \in Y$. We have two cases to consider: $q_{k}<\tilde{q}_{k}$ and $q_{k}>\tilde{q}_{k}$.

Consider first the case $q_{k}>\tilde{q}_{k}$. Assume agent $k$ has the $l$ th higher demand among the demands that constitute the smallest Nash equilibrium. When coalition $S$ deviates and player $k$ decreases her demand two situations can emerge: Either she remains as the agent with the $l$ th higher demand, or she becomes the agent with the $(l-r)$ th demand for some $r=1, \ldots, l-1$.

In the first case it is easy to see that agent $k$ is worse off if the coalition deviates because $q_{k}$ is the smallest equilibrium strategy of player $k$ (this 
implies $u_{k}\left(q_{k}, x_{k}^{D}\left(q_{k}, q_{-k}\right)\right)>u_{k}\left(\tilde{q}_{k}, x_{k}^{D}\left(\tilde{q}_{k}, q_{-k}\right)\right) \quad$ with $\quad x_{k}^{D}\left(\tilde{q}_{k}, q_{-k}\right)=$ $x_{k}^{D}\left(\left(\tilde{q}_{i \in S},(q)_{i \notin S}\right)\right.$ since player $k$ 's payment only depends upon demands larger than her own.

If the second case holds, it implies that there exists $j$ such that $q_{k} \geqslant \tilde{q}_{j}>\tilde{q}_{k}$, where we assume w.l.o.g. $j \in S$. This implies that $u_{k}\left(q_{k}, x_{k}^{D}\left(q_{k}, q_{-k}\right)\right)>u_{k}\left(\tilde{q}_{k}, x_{k}^{D}\left(\tilde{q}_{k}, q_{-k}\right)\right) \geqslant u_{k}\left(\tilde{q}_{k}, x_{k}^{D}\left(\tilde{q}_{k}, \tilde{q}_{j}, q_{-k, j}\right)\right)$. The first inequality is due to the fact that $q_{k}$ is the smallest equilibrium strategy, recall that $q_{k}>\tilde{q}_{k}$, and the last inequality is due to the cross monotonicity property of the decreasing serial rule. In either case, agent $k$ 's utility, $k \in S$, decreases when coalition $S$ deviates.

Consider now the case $q_{k}<\tilde{q}_{k}$. Let $s$ be the second largest element in $Y$, i.e., $\tilde{q}_{s} \geqslant \tilde{q}_{j}$ for all $j \in Y, j \neq k$. Since $q_{s}$ is player $s$ 's smallest Nash equilibrium strategy we know that $u_{s}\left(q_{s}, x_{s}^{D}(\mathbf{q})\right) \geqslant u_{s}\left(\tilde{q}_{s}, x_{s}^{D}\left(\tilde{q}_{s}, q_{-s}\right)\right)$, with strict inequality if $q_{s}>\tilde{q}_{s}$. Because of the cross monotonicity property of the decreasing serial rule the payment of agent $s$ will be bigger with $\tilde{q}_{k}$ than with $q_{k}$, therefore $u_{s}\left(\tilde{q}_{s}, x_{s}^{D}\left(\tilde{q}_{s}, q_{-s}\right)\right)>u_{s}\left(\tilde{q}_{s}, x_{s}^{D}\left(\tilde{q}_{s}, \tilde{q}_{k}, q_{-s, k}\right)\right)$. Hence there is at least a member of $S$ that suffers a decrease in her utility if this coalition deviates. Notice that $u_{s}\left(q_{s}, x_{s}^{D}(\mathbf{q})\right)>u_{s}\left(\tilde{q}_{s}, x_{s}^{D}\left(\tilde{q}_{s}, \tilde{q}_{k}, q_{-s, k}\right)\right)=$ $u_{s}\left(\tilde{q}_{s}, x_{s}^{D}\left(\left(\tilde{q}_{i}\right)_{i \in S},\left(q_{i}\right)_{i \notin S}\right)\right)$, where the last equality is due to the independence of agent $s$ 's cost share with respect to demands lower than her own. This proves that the smallest Nash equilibrium is a strong equilibrium of the decreasing serial game. It is straightforward to see that it is the unique strong equilibrium once we know that it is the Pareto superior equilibrium.

We now show that the smallest Nash equilibrium of the decreasing serial game is the Pareto superior equilibrium. From Theorem 7 in Milgrom and Roberts [2] we know that whenever the players' payoff functions are monotone decreasing in the other players' strategies, then the smallest equilibrium is the Pareto superior equilibrium. The decreasing serial game satisfies this property. Note that for each player an increase in other players' strategies implies an increase in her payment and thus a reduction in her utility. Therefore each player's payoff function is monotone decreasing in the other players' strategies, and thus, our claim holds.

Proposition 2. For $C \in \mathrm{C}$ given and for any $\left(u_{1}, \ldots, u_{n}\right)$ in $\mathscr{D}$, if (AU) holds relative to $C$ then the unique Nash equilibrium of the decreasing serial cost sharing game is a strong equilibrium as well.

Proof. Assume, to the contrary, that $\left(q_{1}^{*}, \ldots, q_{n}^{*}\right)$ is the unique Nash equilibrium of the decreasing serial game but that it is not a strong equilibrium, that is to say, there exists $S \subseteq N$ and $(\tilde{q})_{i \in S}$, with $\tilde{q}_{i} \neq q_{i}^{*}$ for some $i \in S$, such that $u_{j}\left(\tilde{q}, x_{j}^{D}\left((\tilde{q})_{i \in S},\left(q_{i}^{*}\right)_{i \notin S}\right)\right) \geqslant u_{j}\left(q_{j}^{*}, x_{j}^{D}\left(\mathbf{q}^{*}\right)\right)$ for every $j \in S$. 
Let $k$ be the biggest index agent in $S$ using a strategy different from $q_{k}^{*}$. For this agent, it must be true that $u_{k}\left(\tilde{q}_{k}, x_{k}^{D}\left((\tilde{q})_{i \in S},\left(q_{i}^{*}\right)_{i \notin S}\right)\right) \geqslant$ $u_{k}\left(q_{k}^{*}, x_{k}^{D}\left(q^{*}\right)\right)$. The definition of the decreasing serial mechanism implies that $x_{k}^{D}\left((\tilde{q})_{i \in S},\left(q_{i}^{*}\right)_{i \notin S}\right)=x_{k}^{D}\left(\tilde{q}_{k}, q_{-k}^{*}\right)$, and the fact that $q_{k}^{*}$ is the unique Nash equilibrium strategy of agent $k$ implies $u_{k}\left(q_{k}^{*}, x_{k}^{D}\left(\mathbf{q}^{*}\right)\right)>$ $u_{k}\left(\tilde{q}_{k}, x_{k}^{D}\left((\tilde{q})_{i \in S},\left(q_{i}^{*}\right)_{i \notin S}\right)\right)$. Therefore we have:

$$
\begin{aligned}
u_{k}\left(\tilde{q}_{k}, x_{k}^{D}\left((\tilde{q})_{i \in S},\left(q_{i}^{*}\right)_{i \notin S}\right)\right) & \geqslant u_{k}\left(q_{k}^{*}, x_{k}^{D}\left(\left(q_{i}^{*}\right)_{i \in N}\right)\right) \\
& >u_{k}\left(\tilde{q}_{k}, x_{k}^{D}\left((\tilde{q})_{i \leqslant k},\left(q_{i}^{*}\right)_{i>k}\right)\right) .
\end{aligned}
$$

The contradiction proves the claim.

\subsection{Characterization of the Serial Mechanisms}

To conclude this section we provide a characterization of the serial mechanisms within the set of mechanisms in $\Omega$, i.e., within the family of cost sharing mechanisms satisfying the properties of anonymity, smoothness and strict monotonicity. The characterization theorem relies on the strategic and welfare properties of these mechanisms. We fix a cost function in $C$ that it is assumed to be continuously differentiable to ensure the smoothness of the family of cost sharing mechanisms that we consider.

TheOREM 4. Let $C \in \mathrm{C}$ be continuously differentiable and given and let $\xi$ be a cost sharing mechanism in $\Omega$. If either of the two following properties holds:

1. For all $\left(u_{1}, \ldots, u_{n}\right)$ in $\mathscr{D}$, the induced game $G\left(C ; u_{1}, \ldots, u_{n}\right)$ has at most one Nash equilibrium, or

2. For all $\left(u_{1}, \ldots, u_{n}\right)$ in $\mathscr{D}$, every Nash equilibrium of the game $G\left(C ; u_{1}, \ldots, u_{n}\right)$ is a strong equilibrium as well, then $\xi$ is either the decreasing serial mechanism or the increasing one.

If in addition (AU) holds relative to $C$, then properties 1 and 2 are equivalent to $\xi$ being the decreasing serial mechanism.

Proof. See Appendix 2.

\section{APPENDIXES}

\section{Appendix 1}

This appendix contains the proof of the cross monotonicity property of the decreasing serial rule and the proof of Lemma 1. 
Claim. If $q_{j} \leqslant q_{j}^{\prime}$ then $x_{i}^{D}\left(q_{j}, q_{-j}\right) \leqslant x_{i}^{D}\left(q_{j}^{\prime}, q_{-j}\right)$.

Proof. Let us fix $j=i+1$, (for any $j<i$ we know that the payment of agent $i$ will be independent of $\left.q_{j}\right)$ and let $\mathbf{q}=\left(q_{1}, \ldots, q_{i}, q_{i+1}, \ldots, q_{n}\right)$. Denoting by $\beta$ to $q_{i+2}+\cdots+q_{n}$, we have to prove that

$$
\begin{aligned}
C\left(i q_{i}\right. & \left.+q_{i+1}^{\prime}+\beta\right)-\frac{C\left((i+1) q_{i+1}^{\prime}+\beta\right)}{i+1} \\
& \geqslant C\left(i q_{i}+q_{i+1}+\beta\right)-\frac{C\left((i+1) q_{i+1}+\beta\right)}{i+1} .
\end{aligned}
$$

The concavity of $C$ implies

$$
\begin{aligned}
& C\left(i q_{i}+q_{i+1}^{\prime}+\beta\right)-C\left(i q_{i}+q_{i+1}+\beta\right) \\
& \quad \geqslant C\left((i+1) q_{i+1}^{\prime}+\beta\right)-C\left((i+1) q_{i+1}+\beta\right) .
\end{aligned}
$$

Since $1 /(i+1)$ is smaller than one we have

$$
\begin{aligned}
& C\left((i+1) q_{i+1}^{\prime}+\beta\right)-C\left((i+1) q_{i+1}+\beta\right) \\
& \quad \geqslant \frac{C\left((i+1) q_{i+1}^{\prime}+\beta\right)}{i+1}-\frac{C\left((i+1) q_{i+1}+\beta\right)}{i+1} .
\end{aligned}
$$

Hence the claim holds.

Proof of Lemma 1. (i) Given $i<n$, and $q_{i+1}, \ldots, q_{n}$ such that $q_{i+1} \leqslant$ $\cdots \leqslant q_{n}$, the optimistic demands of agent $i$ on the opportunity set $\eta\left(i ; q_{i+1}, \ldots, q_{n}\right)$ are the solutions to the following program:

$$
\begin{aligned}
& \max _{q_{i}} u_{i}\left(q_{i}, x_{i}\right) \\
& \text { s.t. } q_{i} \geqslant 0 \\
& x_{i}=\left\{\begin{array}{lll}
x_{n}^{D}\left(n \cdot q_{i}\right) & \text { if } & q_{i} \geqslant q_{n} \\
x_{n-1}^{D}\left((n-1) \cdot q_{i}, q_{n}\right) & \text { if } & q_{n-1} \leqslant q_{i} \leqslant q_{n} \\
\vdots & & \\
x_{i}^{D}\left(i \cdot q_{i}, q_{i+1}, \ldots, q_{n}\right) & \text { if } & q_{i} \leqslant q_{i+1} .
\end{array}\right.
\end{aligned}
$$


Let, w.l.o.g., $q_{i} \leqslant q_{i+1}$ be a solution to the above program. A solution to this program exists because of (A1). Next we show the uniqueness of the solution by proving that the first-order conditions cannot have more than one solution. The first-order conditions are:

$$
\begin{gathered}
\frac{\partial u_{i}\left(q_{i}, x_{i}\right) / \partial q_{i}}{\partial u_{i}\left(q_{i}, x_{i}\right) / \partial x_{i}}=-C^{\prime}\left(q^{i}\right), \\
x_{i}=x_{i}^{D}\left(i \cdot q_{i}, q_{i+1}, \ldots, q_{n}\right)=\frac{C\left(i q_{i}+q_{i+1}+\cdots+q_{n}\right)-x_{i+1}^{D}-\cdots-x_{n}^{D}}{i} .
\end{gathered}
$$

Equation (2) into (1) implies the following optimality condition:

$$
\frac{\partial u_{i}\left(q_{i}, x_{i}^{D}\left(i \cdot q_{i}, q_{i+1}, \ldots, q_{n}\right)\right) / \partial q_{i}}{\partial u_{i}\left(q_{i}, x_{i}^{D}\left(i \cdot q_{i}, q_{i+1}, \ldots, q_{n}\right)\right) / \partial x_{i}}=-C^{\prime}\left(i q_{i}+q_{i+1}+\cdots+q_{n}\right)
$$

Since the cost function is increasing, Eq. (3) is equivalent to:

$$
\frac{\operatorname{MRS}^{i}\left(q_{i}, x_{i}^{D}\left(i \cdot q_{i}, q_{i+1}, \ldots, q_{n}\right)\right)}{C^{\prime}\left(i q_{i}+q_{i+1}+\cdots+q_{n}\right)}=1
$$

where $q_{i+1}, \ldots, q_{n}$ are fixed.

If the function $g\left(q_{i}\right)=\left(M R S^{i}\left(q_{i}, x_{i}^{D}\left(q_{i}, \ldots, q_{i}, q_{i+1}, \ldots, q_{n}\right)\right) / C^{\prime}\left(i q_{i}+q_{i+1}\right.\right.$ $\left.\left.+\cdots+q_{n}\right)\right)-1$ is strictly decreasing then Eq. (4) cannot have more than one solution. It has at least one solution due to (A1). Condition (AU) implies that $g(\cdot)$ is a decreasing function hence our claim holds.

(ii) Let $q_{j}^{\prime}, q_{i}^{\prime}$ be agents' $j$ and $i$ optimistic demand on $\eta(k$; $\left.q_{i+1}, \ldots, q_{n}\right)$. Let us assume, by way of contradiction, $q_{j}^{\prime} \leqslant q_{i}^{\prime}$ and $q_{j}^{\prime \prime} \geqslant q_{i}^{\prime}$, where $q_{j}^{\prime \prime}$ is agent $j$ 's optimistic demand on the set $\eta\left(i-1 ; q_{i}^{\prime}, q_{i+1}, \ldots, q_{n}\right)$. Thus we have $q_{j}^{\prime} \leqslant q_{i}^{\prime} \leqslant q_{j}^{\prime \prime}$. Since preferences are single-peaked on every possible opportunity set an agent may face, it must be true that $\partial u_{j} / \partial q_{j}<0$ for all $q_{j}>q_{j}^{\prime}$, and $\partial u_{j} / \partial q_{j}>0$ for all $q_{j}<q_{j}^{\prime \prime}$, where $q_{j}^{\prime}=\arg \max _{q} u_{j}\left(q_{j}, x_{j}\right)$ on the set $\eta\left(k ; q_{i+1}, \ldots, q_{n}\right)$ and $q_{j}^{\prime \prime}=\arg \max _{q} u_{j}\left(q_{j}, x_{j}\right)$ on the set $\eta\left(i-1 ; q_{i}^{\prime}, q_{i+1}, \ldots, q_{n}\right)$. This implies that for all $q_{j} \in\left(q_{j}^{\prime}, q_{j}^{\prime \prime}\right)$ the marginal utility of the output, $\partial u_{j} / \partial q_{j}$, must be, at the same time, positive and negative; a contradiction.

The contradiction proves the claim.

\section{Appendix 2}

In this Appendix we provide the proofs of our two main theorems which proofs are closely related. The proofs are also related to some proofs in 
Moulin and Shenker [5]. In particular, we make use of three lemmata that we take from [5]. We here provide the statements of these lemmata for the sake of completeness.

We start by introducing some notations.

We say that a matrix $\left[a_{i, j}\right]$ has a cycle $\left(i_{1}, \ldots, i_{L}\right)$ if the $i_{k}$ element forms a nonrepeating sequence such that $a_{i L, i 1} \neq 0$ and $a_{i k, i k+1} \neq 0$ for all $1 \leqslant k \leqslant L-1$. A matrix with no cycles of length greater than one will be called acyclic. A square acyclic matrix must have an element $j$ such that $a_{i j}=0$ for all $i \neq j$. We will call $j$ a tail element. We will call subtail to any element which breaks a cycle of length smaller than $n$. We will say that a mechanism $\xi$ is acyclic at a point $\mathbf{q} \in \mathscr{R}_{+}^{n}$ if the matrix $\left[\partial \xi_{i} / \partial q_{j}\right]$ is acyclic at that point. We denote the matrix $\left[\partial \xi_{i} / \partial q_{j}\right]$ by $P_{i j}$.

Throughout this appendix we work with two families of cost sharing mechanisms, $\Omega$ and a subset of this family that we denote by $\Pi$. Recall that $\Omega$ is composed of cost sharing mechanisms that satisfy the properties of anonymity, Smoothness and strict monotonicity. $\Pi$ is the subset of mechanisms in $\Omega$ that induce a cost sharing game with at least one Nash equilibrium for the entire domain of convex and monotone preferences.

Lemma 2. Let $\xi$ be a mechanism in $\Omega$. Such mechanism is either the decreasing or the increasing serial mechanism if and only if the matrix $P_{i j}$ is acyclic for all $\mathbf{q} \in \mathscr{R}_{+}^{n}$. Moreover, if $p_{i j} \geqslant 0$ for all $j \neq i$ then $\xi$ is the decreasing serial mechanism, and if $p_{i j} \leqslant 0$ for all $j \neq i$ then $\xi$ is the increasing serial mechanism.

Proof. I first show some characteristics of the acyclic matrices since it will be helpful in order to follow the proof of this lemma.

An acyclic matrix can have no cycles of length greater than one. This implies that a square $(n \times n)$ acyclic matrix must have at least one tail element and $(n-2)$ subtail elements, one for each one of the $(n-2)$ submatrices of dimension $i, i=2, \ldots, n-1$, which are the principal minors associated with the tail element and each of the subtail elements. The next example may help to illustrate this statement.

Consider the following matrix A:

$$
\left(\begin{array}{cccc}
a_{11} & a_{12} & 0 & 0 \\
0 & a_{22} & a_{23} & 0 \\
a_{31} & 0 & a_{33} & 0 \\
a_{41} & a_{42} & a_{43} & a_{44}
\end{array}\right)
$$

Element 4 is a tail for A since $a_{i 4}=0$ for all $i \neq 4$. Thus A has no cycles of length 4 . Nevertheless $A$ is not an acyclic matrix since there exists a cycle 
of length 3 , note that $a_{12}, a_{23}$, and $a_{31}$ are all different from 0 so $\mathrm{A}$ has the cycle $(1,2,3)$. This matrix converts into an acyclic matrix when one assumes $a_{12}=0$. If this were the case then 4 would be the tail element of A, 2 would be the subtail element of the principal minor associated with $a_{44}$, and 3 would be the subtail element of the $2 \times 2$ submatrix that constitutes the principal minor associated with $a_{22}$. Therefore if $\mathrm{A}$ is acyclic then it must have at least one tail element and two subtail elements.

We can now proceed to prove the lemma.

The if part of the lemma holds easily, since the matrix $P_{i j}$ associated with either serial rule is triangular and therefore it is an acyclic matrix.

The only part of the lemma is shown in two steps:

Step 1. In this step we show that the tail element of the matrix $P_{i j}$ has to be either 1 or $n$. We then show that if 1 is the tail element of the matrix of dimension $n, 2$ must be the subtail element of the submatrix of dimension $(n-1)$ associated with $a_{11}, 3$ will be the subtail element of the submatrix of dimension $(n-2)$ associated with $a_{22}$ and so on.

Consider, by way of contradiction, a mechanism $\xi$ in $\Omega$ and an acyclic $P_{i j}$ whose tail element $j$ is different from 1 or $n$.

Since $j$ is a tail element and since budget balance must hold $\left(\sum_{i=1}^{n} \xi_{i}(C ; \mathbf{q})\right.$ $\left.=C\left(\sum_{i=1}^{n} q_{i}\right)\right)$ then $p_{j j}$ must be equal to $C^{\prime}\left(\sum_{i=1}^{n} q_{i}\right)$. Consider now the rows $j-1$ and $j+1$ of $P_{i j}$. Because of acyclicity at least one of the following cases must hold:

1. $p_{j-1, j+1}=0$ and $p_{j+1, j-1} \neq 0$.

2. $p_{j-1, j+1} \neq 0$ and $p_{j+1, j-1}=0$.

3. $p_{j-1, j+1}=p_{j+1, j-1}=0$.

Assume the first one holds. We have $p_{j-1, j}=p_{j-1, j+1}=0$. This implies that agent $j-1$ 's payment does not depend on the demands of agent $j$ or $j+1$. Thus, appealing to anonymity, $p_{j-1, j-1}$ must be equal to $C^{\prime}\left(3 q_{j-1}+\sum_{k \neq j, j-1, j+1} q_{k}\right)$.

At $\mathbf{q}^{*}, \mathbf{q}^{*} \in \mathscr{R}_{+}^{n}$ with $q_{j}^{*}=q_{j-1}^{*} \neq q_{j+1}^{*}$, the value of $p_{j j}$ and $p_{j-1, j-1}$ are:

$$
p_{j-1, j-1}=C^{\prime}\left(3 q_{j-1}^{*}+\sum_{k \neq j, j-1, j+1} q_{k}^{*}\right),
$$

and

$$
p_{j j}=C^{\prime}\left(2 q_{j-1}^{*}+q_{j+1}^{*} \sum_{k \neq j, j-1, j+1} q_{k}^{*}\right)
$$


Now $p_{j j}\left(\mathbf{q}^{*}\right) \neq p_{j-1, j-1}\left(\mathbf{q}^{*}\right)$ implies that $\xi$ is not an anonymous mechanism, contradicting the assumption of $\xi$ in $\Omega$. The same reasoning applies to cases 2 and 3 . The contradiction proves that either 1 or $n$ must be the tail elements of $P_{i j}$.

Let us assume, w.l.o.g., that 1 is the tail element of $P_{i j}$. By a similar reasoning to the one used above it can be shown that 2 has to be the subtail of the submatrix of dimension $n-1$ associated with $p_{11}$. If this were not the case then anonymity would fail at $\mathbf{q}^{\prime}, \mathbf{q}^{\prime}=\left(q_{2}, q_{2}, q_{3}, \ldots, q_{n}\right)$, since $p_{11}\left(\mathbf{q}^{\prime}\right)$ would be different from $p_{22}\left(\mathbf{q}^{\prime}\right)$

Therefore if 1 is the tail element of $P_{i j}$ then $P_{i j}$ must be a triangular superior matrix whose elements in the diagonal are:

$$
p_{j j}=C^{\prime}\left(j q_{j}+\sum_{k>j} q_{k}\right) .
$$

Step 2. We now show that if 1 is the tail element of the matrix $P_{i j}$, recall that $P_{i j}=\left[\partial \xi_{i} / \partial q_{j}\right]$, then $\xi$ must be the decreasing serial mechanism. We start by proving that the elements $p_{i j}$ beneath the diagonal cannot be equal to zero.

Assume instead $p_{i j}=0$ for some $i, j$ beneath the diagonal. This implies that $\xi_{i j}$ does not depend on $q_{j}$, therefore $p_{i i}$ must be equal to $C^{\prime}\left((i+1) q_{i}+\sum_{(k>j)_{k+j}} q_{k}\right)$. But $p_{i i}=C^{\prime}\left(i q_{i}+\sum_{k>i} q_{k}\right)$ since it is an element in the diagonal. The contradiction proves that all $p_{i j}$ beneath the diagonal differ from zero.

To complete the proof we now show that $\xi$ coincides with the decreasing serial mechanism. Consider first $\xi_{n}$. Since $p_{n n}=\partial x_{n} / \partial q_{n}=C^{\prime}\left(n q_{n}\right)$ then $\xi_{n}(C ; q)=C\left(n q_{n}\right) / n+B$. Take $\mathbf{q}^{*}=\left(q_{n}, \ldots, q_{n}\right)$, at $\mathbf{q}^{*}$ we have $\xi_{i}\left(C, \mathbf{q}^{*}\right)=$ $\xi_{n}\left(C, \mathbf{q}^{*}\right)=C\left(n q_{n}\right) / n+B$ for all $i \in N$. Because of budget balance $\sum_{i} \xi_{i}(C ; \mathbf{q})$ has to be equal to $C\left(\sum_{i} q_{i}\right)$. This implies $B=0$ and, hence, $\xi_{n}(C ; \mathbf{q})=x_{n}^{D}(C ; \mathbf{q})$.

Consider next $\xi_{n-1}$. Since $p_{n-1, n-1}=C^{\prime}\left((n-1) q_{n-1}+q_{n}\right)$ then $\xi_{n-1}(C ; \mathbf{q})=\left(C\left((n-1) q_{n-1}+q_{n}\right) /(n-1)\right)+F$. Because of $\xi_{n-1}\left(C, \mathbf{q}^{*}\right)=$ $C\left(n q_{n}\right) / n$ we have that $F=-C\left(n q_{n}\right) / n(n-1)$. Thus $\xi_{n-1}(C ; \mathbf{q})=x_{n-1}^{D}(C ; \mathbf{q})$. Invoking anonymity for the remaining payments, the claim holds.

Thus steps 1 and 2 prove the lemma.

Remark 1. When the cost function is strictly convex Moulin and Shenker [5] have characterized the increasing serial mechanism as the only mechanism that induces a cost sharing game with a unique equilibrium for all preference profiles in $\mathscr{D}$. The lemma above applies also to convex cost functions. Therefore the decreasing sharing mechanism is disqualified in their characterization theorem because, for some demands, it might yield negative cost shares. Moulin and Shenker [5] rule out transfer of inputs 
among agents in their definition of cost sharing mechanisms, so the payments must be non-negative.

Lemma 3. (Claim 2 in Moulin and Shenker [5].) Let $\xi$ be a mechanism in $\Omega$. For every $\mathbf{q} \in \mathscr{R}^{n}{ }_{+}$there exists a set of continuously differentiable utility functions $\left(u_{1}, \ldots, u_{n}\right)$ in $\mathscr{D}$ with $\left(\partial u_{i}\left(q_{i}, \xi_{i}(\mathbf{q})\right) / \partial x_{i}\right) \neq 0$ and $\left(\partial u_{i}\left(q_{i}, \xi_{i}(\mathbf{q})\right) / \partial q_{i}\right) \neq 0$, such that the set of Nash equilibria associated with those $\left(u_{1}, \ldots, u_{n}\right)$ contains $\mathbf{q}$.

Lemma 4. (Lemma 5 in Moulin and Shenker [5].) Let us consider a mechanism $\xi \in \Omega$. If every Nash equilibrium of the cost sharing game induced by $\xi$ is a strong equilibrium as well then the mechanism is acyclic at all $\mathbf{q} \in \mathscr{R}_{+}^{n}$.

Lemma 5. (Lemma 6 in Moulin and Shenker [5].) Consider a mechanism $\xi$ in $\Omega$. If there is at most one Nash equilibrium for every profile $\left(u_{1}, \ldots, u_{n}\right)$ in $\mathscr{D}$, then the mechanism is acyclic at all $\mathbf{q} \in \mathscr{R}^{n}{ }_{+}$.

Lemma 6. Consider a cyclic mechanism $\xi$ in $\Pi$. If for every $\left(u_{1}, \ldots, u_{n}\right)$ in $\mathscr{D}_{\mathcal{N}}$ the game $G\left(C ; u_{1}, \ldots, u_{n}\right)$ has at least one Nash equilibrium then $\xi_{i}$ has to be nonincreasing in $q_{j}$, for all $q_{j} \neq q_{i}$ and for any $i \in N$.

Proof. Fix a $\xi \in \Pi$. Since $\xi$ is a cyclic mechanism then the matrix $P_{i j}(\mathbf{q})$ has a cycle at some point $\mathbf{q} \in \mathscr{R}_{++}^{n}$. Appealing to Lemma 3 we know that there exists a set of continuously differentiable utility functions $\left(u_{1}, \ldots, u_{n}\right)$ in $\mathscr{D}_{\mathcal{N}}$ such that $\mathbf{q}$ is a Nash equilibrium of $G\left(C ; u_{1}, \ldots, u_{n}\right)$. Moreover, because of Lemma 4 we know that for all the agents in the cycle $u_{i}\left(q_{i}+\varepsilon \Delta_{i}, \xi_{i}(\mathbf{q}+\varepsilon \boldsymbol{\Delta})\right)>u_{i}\left(q_{i}, \xi_{i}(\mathbf{q})\right)^{14}$ for each $i$ such that $\Delta_{i} \neq 0$. For these utility functions if the matrix $P_{i j}=\left[\partial \xi_{i} / \partial q_{j}\right]$ has a cycle then the matrix $S_{i j}=\left[\partial u_{i}\left(q_{i}, \xi_{i}(\mathbf{q})\right) / \partial q_{j}\right]$ has a cycle as well. Let $(1, \ldots, L)$ be the shortest cycle of the matrix $S_{i j}$. Since it is the shortest cycle then in the submatrix of dimension $L \times L$ the only elements different from zero will be those of the cycle (any $s_{i i}$ will be zero since $\mathbf{q}$ is a Nash equilibrium). Therefore $\xi_{i}$ is independent of $q_{j}$ for all $j \neq i+1$ and for each $i=1, \ldots, L-1$. Let $i$ be an agent in the cycle. We now show that if $\Delta_{i} \neq 0$ then $\Delta_{i}$ must be positive. The acyclicity of $\xi$ implies that for every $\varepsilon$ we can find a preference profile $\left(v_{1}, \ldots, v_{n}\right)$ in $\mathscr{D}_{N}$ such that for this profile $(\mathbf{q})$ and $(\mathbf{q}+\varepsilon \mathbf{\Delta})$ are Nash equilibria of $G\left(C ; v_{1}, \ldots, v_{n}\right)$. For those agents that are not in the cycle $v_{i}$ must be such that $q_{i}=\operatorname{argmax}\left[v_{i}\left(z_{i}, \xi_{i}\left(z_{i}, q_{-i}\right)\right)\right]$ and

\footnotetext{
${ }^{14}$ Note that Lemmata 3 and 4 refer to preferences in $\mathscr{D}$. Nevertheless it is straightforward to show (this is clear from the proofs) that the statement of the lemmata holds if we consider preferences in $\mathscr{D}_{\mathcal{N}}$.
} 
$q_{i}=\operatorname{argmax}\left[v_{i}\left(z_{i}, \xi_{i}\left(z_{i},(q+\varepsilon \Delta)_{-i}\right)\right)\right]$. Such $v_{i}$ does exist by the strict monotonicity assumption on the preferences and on the cost sharing mechanism.

For the agents in the cycle, take $v_{i}=u_{i}$. Now the binormality of the preferences and the fact that $u_{i}\left(q_{i}+\varepsilon \Delta_{i}, \xi_{i}(\mathbf{q}+\varepsilon \mathbf{\Delta})\right)>u_{i}\left(q_{i}, \xi_{i}(\mathbf{q})\right)$ imply the result. $\Delta_{i}$ must be positive since the MRS has to be steeper at $\left(q_{i}, \xi_{i}\left(q_{i}, q_{-i}\right)\right)$ than at $\left(q_{i}, \xi_{i}\left(q_{i},(q+\varepsilon \Delta)_{-i}\right)\right)$. If $\Delta_{i}>0$ and $u_{i}\left(q_{i}+\varepsilon \Delta_{i}\right.$, $\left.\xi_{i}(\mathbf{q}+\varepsilon \mathbf{\Delta})\right)>u_{i}\left(q_{i}, \xi_{i}(\mathbf{q})\right)$ then $\xi_{i}$ has to be nonincreasing in $q_{j}$, for all $q_{j} \neq q_{i}$ and for any $i=1, \ldots, L-1$. Now anonymity ensures that the result has to hold for the agents not in the cycle. Lemma 3 renders this conclusion applicable to all $\mathbf{q}$. This completes the proof of the lemma.

Lemma 7. Consider a cyclic mechanism $\xi \in \Pi$. If for every $\left(u_{1}, \ldots, u_{n}\right)$ in $\mathscr{D}_{\mathscr{f}}$ the game $G\left(C ; u_{1}, \ldots, u_{n}\right)$ has at least one Nash equilibrium then $\xi_{i}$ has to be nondecreasing in $q_{j}$, for all $q_{j} \neq q_{i}$ and for any $i \in N$.

Proof. The proof is very similar to the one in previous lemma so it is omitted.

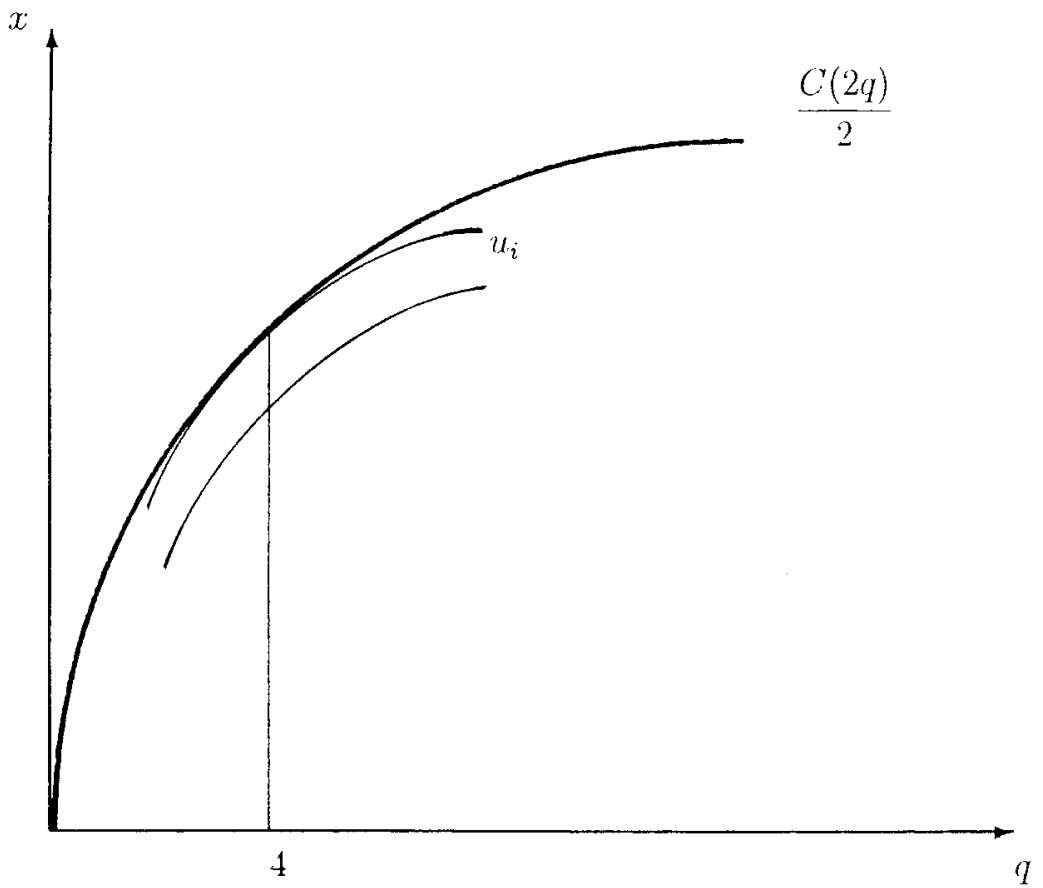

FIG. 1. Agent $i$ 's optimistic demands. 
Proof of Theorem 4. The theorem follows from Lemmata 2, 3, 4, and 5. Theorem 3 and Proposition 2 show that if (AU) holds relative to $C$ then the decreasing serial game has a unique equilibrium which is strong

Proof of Theorem 1. Let $\xi$ be a cyclic mechanism in $\Pi$. Appealing to Lemma 6, $\xi_{i}$ has to be nonincreasing in $q_{j}$ for all $q_{j} \neq q_{i}$ and for any $i \in N$; while by Lemma 7 we know that $\xi_{i}$ has to be decreasing in $q_{j}$ for all $q_{j} \neq q_{i}$. Hence, it must be the case that $\xi_{i}$ does not depend upon $q_{j}$. But this implies that the mechanism is not anonymous but dictatorial contrary to the assumption that $\xi$ belongs to $\Pi$.

Assume now that $\xi$ is an acyclic mechanism in $\Omega$. Appealing to Lemma 2 we can conclude that it has to be either the decreasing serial rule or the increasing serial rule. For the increasing serial rule Moulin [7] provides an example of a preferences profile in $\mathscr{D}$ for which the game induced by the increasing serial rule fails existence of a Nash equilibrium. It is straightforward to adapt his example for the decreasing serial rule. Instead we now provide a graphical example for a decreasing serial game with two agents for which the best reply correspondences are not convex-valued and

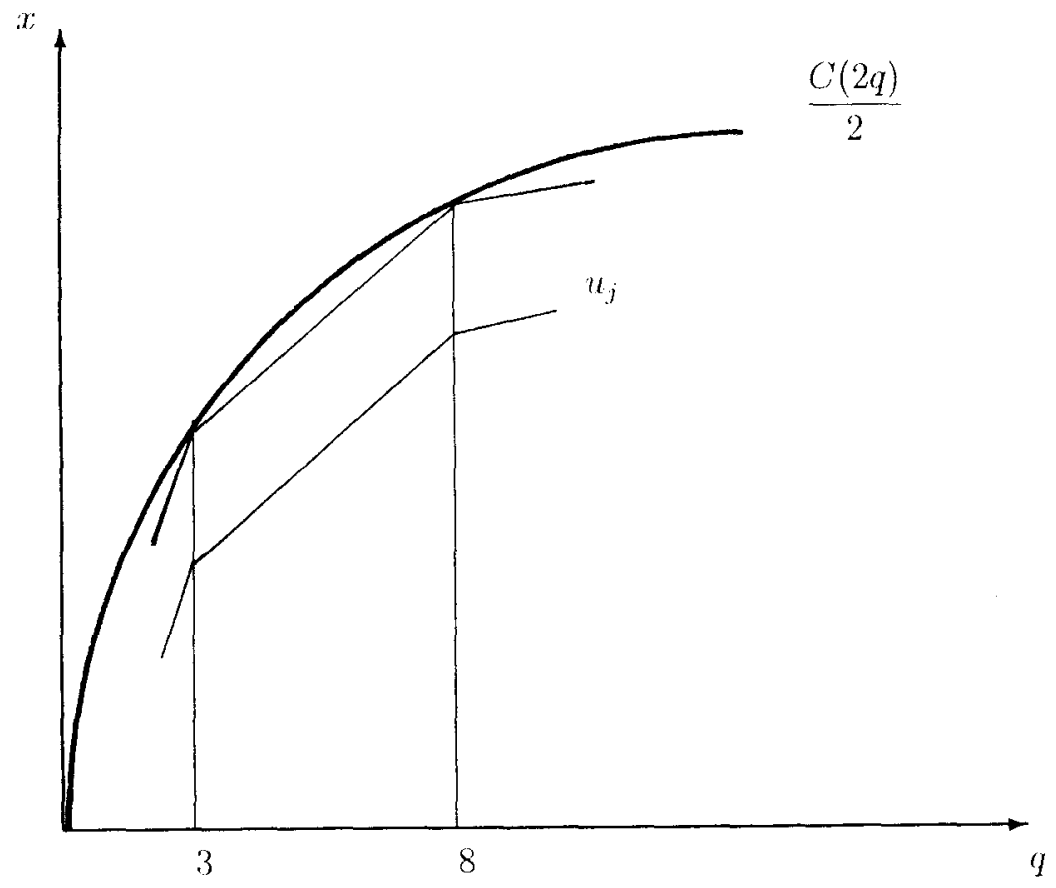

FIG. 2. Agent $j$ 's optimistic demands. 


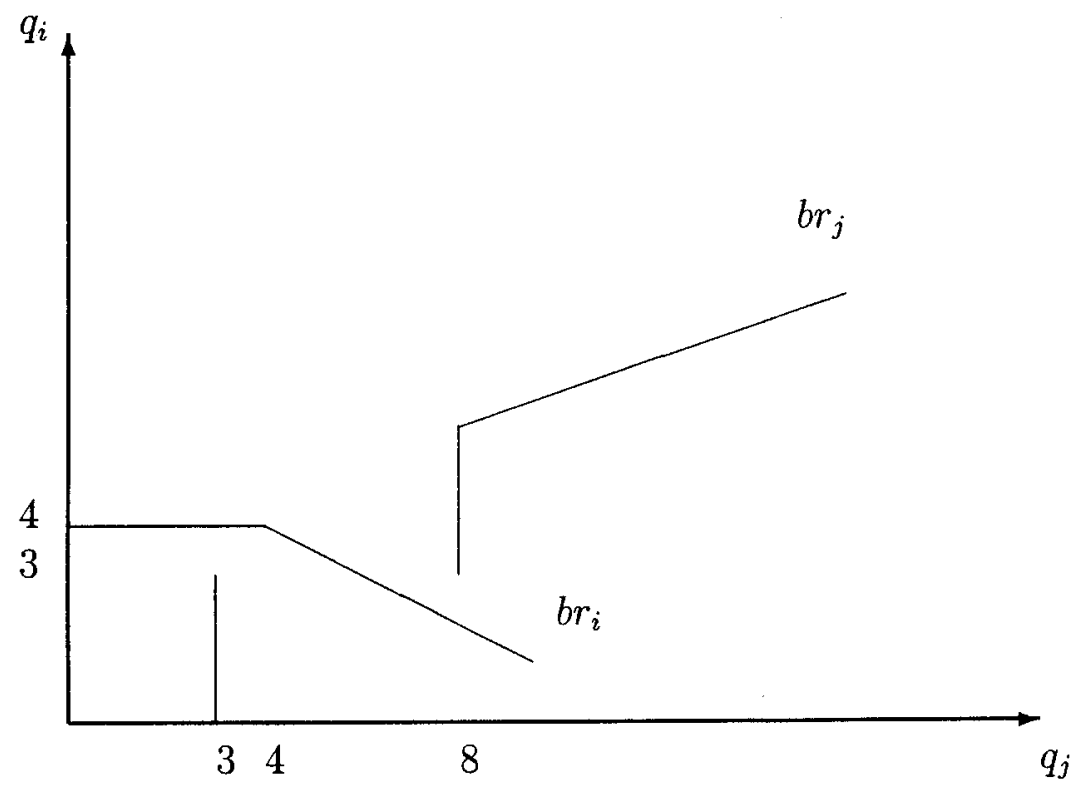

FIG. 3. Best replies.

they do not cross. Figures 1-3 show that the decreasing serial game fails existence of equilibrium for some preferences profile in $\mathscr{D}$, as well.

Thus we can conclude that $I T$ is empty as we wanted to show.

\section{ACKNOWLEDGMENTS}

I am very grateful to Hervé Moulin, Salvador Barberà, Xavier Vives, Debraj Ray, Sandeep Ray, Diego Moreno, the associate editor and two anonymous referees and especially Jordi Massó for many helpful comments. Financial support from Generalitat de Catalunya and grant PB92-0590 from la Dirección General de Investigación Científica y Técnica is gratefully acknowledged.

\section{REFERENCES}

1. Y. Balasko and $\mathrm{K}$. Shell, The overlapping generation model I: the case of pure exchange without money, J. Econ. Theory 26 (1980), 281-306.

2. P. Milgrom and K. Roberts, Adaptative and sophisticated learning in normal form games, Games Econ. Behav. 3 (1991), 82-100.

3. P. Milgrom and K. Roberts, Rationalizability, learning, and equilibrium in games with strategic complementarities, Econometrica 58 (1990), 1255-1277.

4. P. Milgrom and C. Shannon, Monotone comparative statics, Econometrica 62 (1994), $157-180$. 
5. H. Moulin and S. Shenker, Serial cost sharing, Econometrica 60 (1992), 1009-1037.

6. H. Moulin and S. Shenker, Average cost pricing versus serial cost sharing: an axiomatic comparison, J. Econ. Theory 64 (1994), 178-201.

7. H. Moulin, Cost sharing under increasing returns to scale: a comparison of simple mechanisms, Games Econ. Behav. 13 (1996), 225-251.

8. H. Moulin, Welfare bounds in the cooperative production problem, Games Econ. Behav. 4 (1992), 373-401.

9. H. Moulin and A. Watts, Two versions of the tragedy of the commons, Econ. Design, forthcoming.

10. M. Quinzii, Core with increasing returns, J. Econ. Theory 28 (1982), 32-50.

11. M. A. Satterthwaite and H. Sonnenschein, Strategy-proof mechanisms at differentiable points, Rev. Econ. Stud. 48 (1981), 587-597.

12. X. Vives, Nash equilibrium with strategic complementarities, J. Math. Econ. 19 (1990), 305-321.

13. A. Watts, On the uniqueness of the equilibrium in cournot oligopoly and other games, Games Econ. Behav. 13 (1996), 269-285. 